VOL. 8 (1973), 205-209.

\title{
A characterization of weak projectability
}

\section{J.B. Miller}

For a commutative $l$-group $G$ with carrier lattice $\subseteq, G$ is weakly projectable iff the proper prime filters of $\underline{\underline{C}}$ are all maximal filters.

1.

A number of characterizations are known for the property of weak projectability. For example (in the terminology and notation set out below), each of the following properties has been shown to be separately equivalent to weak projectability, for a commutative $z$-group $G$ :

(a) every proper $P_{t}$-ideal of $G$ is minimal prime (Spirason and Strzelecki [5]);

(b) every proper replete $Z$-ideal of $G$ is the intersection of all minimal prime $Z$-ideals containing it (Spirason [4], p. 4-2);

(c) the space $\underline{y}$ of all proper $P_{t}$-ideals is Hausdorff in its hullkernel topology (Davis [1]);

(d) $\underline{\mathrm{H}}$ is a relatively complemented lattice (Davis [1]).

Some other characterizations are given by Spirason in [4]. Speed [3] has shown that if $G$ has a weak unit, then $G$ is weakly projectable iff for every $x \geq 0$ there exists $y \geq 0$ for which $x^{12}=y^{1}$. In this note we give a further characterization.

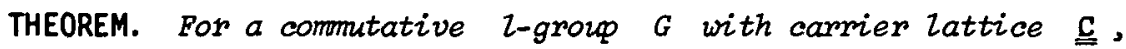

Received 24 October 1972. 
$G$ is weakly projectable iff the proper prime filters of $\underline{\mathrm{C}}$ are all maximal filters.

\section{Terminology, notation and basic results}

An 2 -group $G$ is a partially-ordered group which is also a lattice with respect to the ordering. $G$ will be assumed commutative throughout this note; we write the operations as $\leq, \wedge, \vee$. An $Z$-ideal of $G$ is an order-convex subgroup which is also a sublattice. An $l$-ideal $L$ is prime if $x \wedge y \in L$ implies either $x \in L$ or $y \in L . L$ is minimal in the class of all proper prime $z$-ideals if further $x^{\perp} \pm L$ for all $x \in L$.

For any subset $A \subseteq G, A^{L}$ denotes the polar set $\{x:|x| \wedge|a|=0$ for all $a \in A\}$; for $a \in G, a^{\perp}$ means $\{a\}^{\perp}$. Every polar $A^{+}$is an $Z$-ideal. The set $\underline{\underline{B}}=\left\{A^{+}: A \subseteq G\right\}$ of all polars is a boolean lattice with respect to inclusion. We write $M$ for the set of all maximal ideals of $\underline{\underline{B}}$.

$$
\begin{aligned}
& \text { For } x \geq 0 \text { in } G \text {, the carrier of } x \text { is } \\
& \qquad \hat{x}=\left\{u: u \geq 0, u^{\perp}=x^{\perp}\right\} .
\end{aligned}
$$

The carriers are pairwise disjoint subsets of $G$. $\{\hat{x}: x \geq 0\}$ of all carriers; ordered by

$$
\hat{x} \leq \hat{y} \Leftrightarrow x^{\perp} \supseteq y^{\perp} \Longleftrightarrow x^{11} \subseteq y^{11},
$$

$\underline{\underline{\mathrm{C}}}$ is a distributive disfunctive lattice with zero $\hat{0}=\{0\}$, and $\underline{\underline{\mathrm{C}}}$ has a greatest element, namely the set

$$
\underline{\underline{w}}=\left\{w>0: w^{\perp}=(0)\right\}
$$

of all weak units of $G$, iff $\underline{\underline{w}} \neq \emptyset$. Since $\underline{\underline{C}}$ is distributive its maximal filters are all prime filters: we are concerned in this note with the converse property.

An $Z$-ideal $L$ is called replete if $x \in L$ and $|y|^{\wedge}=|x|^{\wedge}$ imply $y \in L$. For any $z \in G, z^{1+}$ is the smallest replete $l$-ideal containing $z$. Thus an $l$-ideal $L$ is replete iff $x \in L$ implies $x^{L L} \subseteq L$. For the elementary properties of polars, carriers and $l$-ideals see for example 
Fuchs [2], Chapter V.

The concept of weak projectability is due to Strzelecki: $G$ is called weakly projectable if for every pair $x, y \in G$ there exists an element $u \in x^{L}$ such that $y \in(|u|+|x|)^{\perp \perp} . G$ is called Stone if for every $a \in G, \quad a^{\perp} \oplus a^{1 \perp}=G$. Every Stone $z$-group is weakly projectable [5]. Every $\sigma$-complete $l$-group is weakly projectable, since every $\sigma$-complete 2-group is Stone [3]. On the other hand, $C[0,1]$ is weakly projectable [5], but not Stone [3]. Strzelecki gives in [6] an example of a nonweakly projectable commutative $l$-group.

For any maximal ideal $t \in \underline{\underline{M}}$ write

$$
P_{t}=\left\{x \in G: x^{11} \in t\right\} \text {. }
$$

$P_{t}$ is a prime $Z$-ideal of $G$. Such ideals are called ' $P_{t}$-ideals' by Veksler [7]; let $\underline{\mathrm{V}}$ denote the set $\left\{P_{t}: P_{t} \neq G, t \in \underline{\underline{M}}\right\}$ of all proper

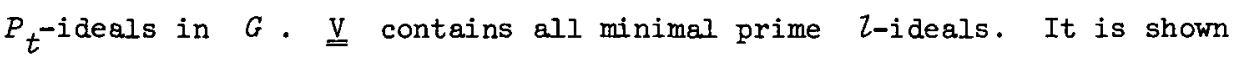
in [5] that a proper prime l-ideal $I$ of $G$ belongs to $\underline{\mathrm{V}}$ iff $I$ is replete. For any $a \in G$ write $V(a)=\left\{P_{t} \in \underline{\underline{V}}: a \notin P_{t}\right\}$. The $\underline{\underline{H}}$ mentioned in (d) above is the lattice $\{V(a): a \in G\}$, under inclusion.

3.

With these results, the proof of the theorem is easy. First, assume $G$ weakly projectable, and let $Z$ be any proper prime filter in the

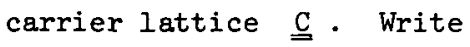

$$
L_{Z}=\left\{x \in G:|x|^{\wedge} \mid z\right\} .
$$

Then $L_{Z}$ is a proper prime $z$-ideal of $G$. Clearly if $x \in L_{Z}$ then $|x|^{\wedge} \subseteq L_{Z}$, so $L_{Z}$ is replete. So by the result quoted above, $L_{Z} \in \underline{\underline{V}}$. By the characterization (a), $L_{Z}$ is minimal prime. That is, for all $x \in L_{z}$ there exists $y \equiv L_{z}$ such that $|x| \wedge|y|=0$; this is equivalent to the statement: for al $|x|^{\wedge} \mid z$ there exists $|y|^{\wedge} \in Z$ such that $|x|^{\wedge} \wedge|y|^{\wedge}=\hat{0}$. But this asserts that $z$ is a maximal filter. 
Conversely, assume that every prime filter in $\underline{\underline{C}}$ is maximal, let $t \in \underline{\underline{M}}$ and consider $P_{t}$. If there exists a prime filter $Z$ in $\underline{\mathrm{C}}$ such that $L_{Z}=P_{t}$ then, $L_{Z}$ being minimal prime since $Z$ is maximal, every element $P_{t}$ of $\underline{\underline{V}}$ is minimal prime and (a) shows that $G$ is weakly projectable. Hence it remains to find $Z$. Write

$$
y=\left\{\hat{y} \in \underline{C}: y^{11} \epsilon t\right\}, z=\underline{C} \backslash y .
$$

It can be verified that $y$ is a prime ideal, so $Z$ is a prime filter; and

$$
\left.x \in L_{Z} \Leftrightarrow|x|^{\wedge}|z \Leftrightarrow| x\right|^{\wedge} \in Y \Leftrightarrow x^{\perp \perp} \in t \Longleftrightarrow x \in P_{t},
$$

so $L_{Z}=P_{t}$, as required.

4.

It is well known that in any boolean lattice a filter is maximal iff it is prime. There exist weakly projectable $l$-groups without weak units, for which therefore $\underline{\underline{c}}$ is not boolean although all its prime filters are maximal. For an example, which moreover is not Stone, take $G$ to be the sublattice subgroup of $C[0,1]$ consisting of all continuous functions on $[0,1]$ which vanish on some neighbourhood of 0 .

If the carrier lattice $\underline{\underline{C}}$ of $G$ is finite, then $\underline{\underline{C}}$ is a boolean lattice ( $P$. Jaffard; see Fuchs [2], p. 82). Thus

COROLLARY. If an 2 -group $G$ has a finite carrier lattice, then $G$ is weakly projectable.

\section{References}

[1] G. Davis, "Orthogonality relations on abelian groups", J. Austral. Math. Soc. (to appear).

[2] L. Fuchs, Partially ordered algebraic systems (Pergamon, Oxford, London, New York, Paris, 1963).

[3] T.P. Speed, "On commutative $Z$-groups", (to appear). 
[4] G.T. Spirason, "W-l-groups", (PhD thesis, Monash University, 1972).

[5] G.T. Spirason and E. Strzelecki, "A note on $P_{t}$-ideals", J. Austral. Math. Soc. 14 (1972), 304-310.

[6] E. Strzelecki, "An example of a non-weakly-projectable Banach lattice", J. Austral. Math. Soc. (to appear).

[7] A.1. Veksler, "Linear spaces with disjoint elements and their conversion into vector lattices", Leningrad. Gos. Ped. Inst. Učen. Zap. 328 (1967), 19-43.

Department of Mathematics,

Monash University,

Clayton,

victoria. 